\title{
Competition Among Flow, Dissolution, and Precipitation in Porous Media
}

\begin{abstract}
A theoretical and experimental study has been carried out on flow, dissolution, and precipitation in porous media. Flow experiments were performed on linear carbonate cores using acidic ferric chloride solutions. Dissolution of the carbonate by the acid causes an increase in the solution $\mathrm{pH}$, thereby precipitating ferric hydroxide. This precipitate plugs up the pore throats in the medium and increases the resistance to fluid flow. Fluctuations in the permeability ratio were observed during core flood experiments, confirming the competition between channel formation due to dissolution and pore plugging due to precipitation. The evolution of the pore structure was characterized by Wood's metal castings.

A network model has also been developed to describe flow and reaction in porous media. The model was used to simulate the ferric chloride system, and pressure oscillations predicted by the model show identical trends to those observed experimentally. Additionally, the evolution of pores in the network were graphically represented.
\end{abstract}

\section{Introduction}

The current research focuses on flow, dissolution, and precipitation in porous media. This study is of general fundamental interest, since it involves an understanding of reaction kinetics, fines migration, and transport phenomenon. Furthermore, when these processes occur in disordered media predicting their behavior can be an intellectual challenge. In addition, this work finds application in diagenesis, enhanced oil recovery, and geothermal/geochemical applications (Axtmann and Peck, 1976; Hoffman, 1975; Reistad et al., 1978).

A common technique used to reduce the resistance to fluid flow during oil well maintenance procedures and in enhanced oil recovery is the injection of acid into the formation to dissolve part of the porous formation and thereby enlarge the pore space. However, some of the dissolution products can precipitate as the acid is consumed and can plug up the pore throats in the medium thus increasing the resistance to fluid flow. Two types of precipitates commonly observed are silicates and iron hydroxides/oxides. Recent work, Crowe (1986), has shown that precipitation of silica from spent $\mathrm{HF}$ acid is not a major concern as it causes only a small reduction in the permeability. Deposition of iron hydroxides/oxides, however, can cause drastic formation damage if excessive $\mathrm{Fe}^{3+}$ ions are taken into the solution during matrix acidizing. Normally, $\mathrm{Fe}^{3+}$ and $\mathrm{Fe}^{2+}$ can both enter the fiuid stream. The five most common mineral sources of iron in oil bearing formations are pyrrhotite $(\mathrm{FeS})$, pyrite $\left(\mathrm{FeS}_{2}\right)$, hematite $\left(\mathrm{Fe}_{2} \mathrm{O}_{3}\right)$, magnetite $\left(\mathrm{Fe}_{3} \mathrm{O}_{4}\right)$, and siderite $\left(\mathrm{FeCO}_{3}\right)$. For example, the dissolution of hematite by $\mathrm{HCl}$ generates ferric chloride that is followed by precipitation.

$$
\begin{aligned}
\mathrm{Fe}_{2} \mathrm{O}_{3}+\mathrm{CaCO}_{3}+8 \mathrm{HCl}-\cdots & \mathrm{CaCl}_{2} \\
& +2 \mathrm{FeCl}_{3}+\mathrm{CO}_{2}+4 \mathrm{H}_{2} \mathrm{O}
\end{aligned}
$$

While Smith et al. (1968) have shown the ratio of ferrous ion to ferric ion in the reservoir to be about $5: 1$, ferric ion precipitation is potentially more damaging since it can precipitate at $\mathrm{pH}$ of about 2-3 (McMennamy).

\section{Summary of previous models}

Several models for flow and reaction in porous media exist in the literature. A comprehensive review of these and other models is presented in Fogler and Rege (1985). Primary among these models are those by Lund and Fogler (1976) and by Walsh et al. (1984). Other models such as those developed by Cussler (1982), Sahimi and Tsotsis $(1985,1987,1988)$, Shah and Ot- 
tino (1987), Yu and Sotirchos (1987), and Too et al. (1986) consider reaction systems in the absence of convective fluid flow (i.e., diffusion only) and are therefore not described here.

The model developed by Lund and Fogler (1976), and later modified by Hekim and Fogler (1980), simulates flow and dissolution in reaction-rate-limited systems-sandstone acidization with $\mathrm{HF} / \mathrm{HCl}$ acid. Mass balances for the aqueous and mineral species are written assuming finite rates of dissolution. These equations are then solved using method of characteristics or numerical techniques. The model can predict the concentration profiles of the aqueous and mineral species as a function of time and distance, as well as the time for "breakthrough" to occur. Breakthrough is defined as the reaction front, which is the region where the derivative of the acid concentration w.r.t. the axial distance is large, reaching the exit end of the core. In general, a large reduction in the pressure drop is also observed at breakthrough.

Two important dimensionless quantities characterizing the dissolution process emerged from this work-the Damköhler number and the acid capacity number. The Damköhler number, which is the ratio of the rate of reactant consumption by mineral to the rate of convective transport of the reactant, determines the shape of the reaction front. The acid capacity number, which is the ratio of the fluid reactant available in the pore space to the reactant needed to dissolve all of the mineral accessible per unit volume of porous medium, determines the velocity of the reaction front.

The predicted change in the mineral concentration is used to determine the change in the porosity; this change in porosity is then related to the local permeability, $k$, by a single parameter $\beta$

$$
k=\exp (\beta . \Delta \phi)
$$

The overall permeability is then determined by

$$
K=\frac{L}{\int_{0}^{L} d x / k}
$$

where $K$ is the overall permeability, $L$ the length of the core, and $k$ the local permeability. Eq. $1 \mathrm{~b}$ is Darcy's law for single-phase flow for permeabilities linearly in series. Model predictions showed excellent agreement with experimental data over a wide variety of conditions. While the model developed by Lund and Fogler is a good model for acidization of reaction-rate-limited systems, it does not consider precipitation of any salts which can cause formation damage.

A geochemical simulator developed by Walsh et al. (1984), and later modified by Bryant et al. (1986, 1987), Novak et al. (1988), and Hwang et al. (1988), considers a special class of reacting systems, in which the reactions occur infinitely fast. As such all components can, therefore, be considered to be in equilibrium with each other. The mass balance equations for flow, dissolution, and precipitation (see review by Fogler and Rege, 1985) are solved numerically subject to equilibrium constraints. The solution is presented in the form of characteristic waves traversing the porous medium; each wave has a specific velocity and separates two regions of fixed compositions. Changes in mineral composition are then related to the overall permeability using equations similar to those postulated by Lund and Fogler
(1976). The advantage of considering equilibrium constraints is that reacting systems with a large number of components can be considered. A major limitation of this equilibrium model is that it cannot model systems where the components have finite rates of reaction. Furthermore, the assumption of equilibrium does not permit consideration of supersaturation or finite rates of precipitation.

A new model for studying flow and dissolution in carbonates has been recently developed by Hoefner and Fogler (1988). This model is based on the network model representation of the porous medium. Here the flow paths within the porous medium are represented by interconnected bonds or pore throats. The nodes represent the pore spaces or points of mixing. Acid was injected into the network and reaction occurred in the pore throats. The effects of Damköhler number on the pore evolution and permeability breakthrough were extensively studied using the network model for both reaction-rate-limited and mass-transfer-limited regimes. Excellent qualitative agreement was obtained between model predictions and experimental data.

The goal of this study is to develop a general model which can predict the conditions when precipitation occurs, the structure of the channels evolved, and the change in the permeability of the formation due to the dissolution and precipitation of mineral salts.

\section{Experimental Study}

To investigate dissolution and precipitation reactions, ferric chloride was selected as the reacting fluid and limestone as the porous medium. This model system mimics the matrix acidization of limestone formations with hydrochloric acid in which the acid dissolves iron oxide or iron hydroxide scale present in the piping. The acidic solution entering the formation contains ferric ions and the reaction equilibria for this system are shown in Table 1 (Butler, 1964). The $\mathrm{FeCl}_{3} / \mathrm{HCl}$ and carbonate system is also a good model system because:

i) The $\mathrm{FeCl}_{3}$ solution acts like a retarded acid and penetrates into the carbonate core, and causes precipitation to occur deep in the core sample (and not at the face).

ii) The carbonate core has few impurities and is therefore a clean system.

iii) For carbonate systems, the Wood's metal castings technique (Hoefner and Fogler, 1988) along with the neutron radiography (Jasti et al., 1987) can be used to study the structure of the evolved channels due to dissolution and precipitation.

Batch experiments were performed to determine the $\mathrm{pH}$ changes in a solution of $\mathrm{FeCl}_{3} / \mathrm{HCl}$ when known amounts of cal-

\section{Table 1. Ionic Equilibria for Ferric Chloride and Carbonate System}

$$
\begin{aligned}
& \text { Ferric chloride in aqueous solution: } \\
& \mathrm{Fe}^{+3}+\mathrm{H}_{2} \mathrm{O}=\mathrm{FeOH} \mathrm{H}^{++}+\mathrm{H}^{+} \\
& \mathrm{FeOH}^{++}+\mathrm{H}_{2} \mathrm{O}=\mathrm{Fe}(\mathrm{OH})_{2}^{++}+\mathrm{H}^{+} \quad \text { Acidic } \\
& 2 \mathrm{Fe}^{+3}+2 \mathrm{H}_{2} \mathrm{O}=\mathrm{Fe} 2(\mathrm{OH})_{2}^{+4}+2 \mathrm{H}^{+} \\
& \text {Reaction of acidic media and calcium carbonate } \\
& \mathrm{CaCO}_{3}+2 \mathrm{H}^{+}=\mathrm{Ca}^{++}+\mathrm{CO}_{2}+\mathrm{H}_{2} \mathrm{O} \\
& \text { Precipitation of ferric hydroxide occurs when: } \\
& \frac{\left[\mathrm{Fe}^{+3}\right]}{\left[\mathrm{H}^{+}\right]^{3}}>9.1 \times 10^{3} \\
& \mathrm{Fe}^{+3}+3 \mathrm{H}_{2} \mathrm{O}=\mathrm{Fe}(\mathrm{OH})_{3} \downarrow+3 \mathrm{H}^{+} \\
& \quad \text { Precipitate }
\end{aligned}
$$


cium carbonate were added to it. Data for two different initial $\mathrm{FeCl}_{3}: \mathrm{HCl}$ ratios are shown in Figure 1. Initially, the $\mathrm{H}^{+}$concentration decreases steadily and then stabilizes. Further addition of calcium carbonate, however, causes a large amount of precipitation and the hydrogen ion concentration decreases rapidly. The trends shown in Figure 1 can also be estimated using mass and charge balances based on the equilibria shown in Table 1. These estimated values are shown as bold lines in Figure 1 and they agree well with the experimental data.

The $\mathrm{Fe}^{3+}$ concentration as a function of dissolved $\mathrm{Ca}^{2+}$ concentration is shown in Figure 2. This curve was estimated using mass and charge balances for a $0.75 \mathrm{M}$ solution of ferric chloride in which calcium carbonate was dissolved.

\section{Coreflood experiments}

The purpose of the coreflood experiments was to observe the permeability response and the pore evolution, when flow, dissolution, and precipitation occur in porous media. Experiments were performed with Texas Cream Chalk (limestone) cores of $0.038 \mathrm{~m}$ diameter and $0.089 \mathrm{~m}$ length. The initial permeability of these cores was in the 10-30 millidarcy range $(0.01-0.03$ $\mu \mathrm{m}^{2}$ ). A schematic of the experimental apparatus is shown in Figure 3. Mixtures of ferric chloride solution and hydrochloric acid were injected into the cores at various flow rates. These solutions, which act as retarded acids, dissolve parts of the formation generating large channels called wormholes. The Wood's metal casting technique was used to observe the structure of these wormholes.

During a typical run acid is consumed through the dissolution of the carbonate rock; as the $\mathrm{pH}$ increases the iron in solution tends to form ferric hydroxide precipitate (Table 1). The amount of precipitate formed and the regions where it deposits control the extent of permeability decline or formation damage. This in turn is controlled by the flow rate or the Damköhler number. Eventually, a dominant channel reaches the core outlet and the pressure drop across the core drops rapidly resulting in breakthrough.

\section{Effect of flow rate or Damköhler number}

Dissolution processes have been previously characterized in terms of the Damköhler number. The Damköhler number for precipitation is also important in our studies, since it controls the

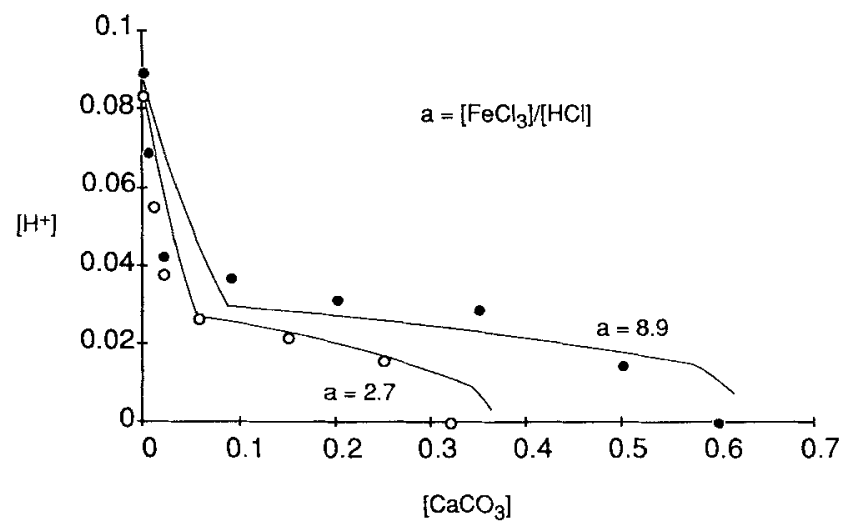

Figure 1. Variation of hydrogen ion concentration with amount of calcium dissolved, for two ferric chloride/ $\mathrm{HCl}$ mixtures.

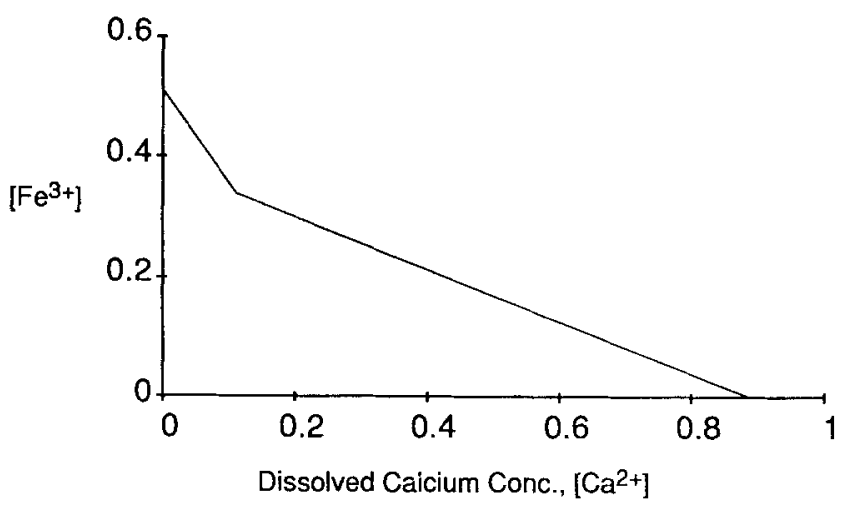

Figure 2. Ferric ion concentration as a function of dissolved calcium.

extent and location of precipitate formation. Solids precipitate from a saturated solution after a certain time for nucleation (induction) has elapsed. This is followed by growth and deposition. At relatively high flow rates, the reactants travel further into the porous medium before deposition takes place. At the same time, the wormhole advances rapidly at high flow rates (Hoefner, 1987). Relatively high flow rates are, therefore, expected to cause relatively less damage. The inverse argument can be applied to low flow rates.

Experiments with the ferric chloride and carbonate system show that the Damköhler numbers for dissolution and precipitation are indeed very important in governing the extent of formation damage. Figure 4 shows the overall core permeability response for different values of the flow rate or the Damköhler number. For relatively low flow rates, the permeability declined to about $0.05-0.01$ of its initial value. Increasing the flow rate (i.e., decreasing the Damköhler number) reduced the extent of permeability decline and the number of pore volumes for breakthrough. More interesting, however, are the pressure fluctuations observed in the different runs; these fluctuations are absent in carbonate acidizing with only $\mathrm{HCl}$. Experiments show that increasing the flow rate reduces the frequency of the fluctuations for a fixed length of the core. For example, in the run performed at $1.39 \times 10^{-9} \mathrm{~m}^{3} / \mathrm{s}(5 \mathrm{~mL} / \mathrm{h})$ approximately 15 fluctuations were observed during the injection of five pore volumes; for the same number of injected pore volumes the $1.1 \times 10^{-8}$ $\mathrm{m}^{3} / \mathrm{s}(40 \mathrm{~mL} / \mathrm{h})$ run exhibited only five fluctuations. The dissolution front for the $5.33 \times 10^{-8} \mathrm{~m}^{3} / \mathrm{s}(192 \mathrm{~mL} / \mathrm{h})$ run broke through without any fluctuations. The explanation for the reduced frequency with increasing flow rate is as follows.

Relatively low flow rates cause deposition of the precipitate to occur only a small distance ahead of the dissolution front (or the advancing wormhole tip). Initially, precipitate formation and deposition occur in the flow paths carrying high flow rates, i.e., paths offering the least resistance to flow. This deposition leads to an increase in the fiow resistance through these paths causing flow diversion to paths which initially carried lower flow rates. As these smaller paths grow past the precipitate zone the resistance to flow is reduced considerably leading to a sudden increase in the permeability response curve shown in Figure 4. For the case of low flow rates, the fluid residence time is high; this results in the formation of a relatively large number of precipitation zones leading to a higher frequency of pressure fluctuations. Eventually most of the fluid is forced to flow through the relatively few unblocked channels at high interstitial veloci- 


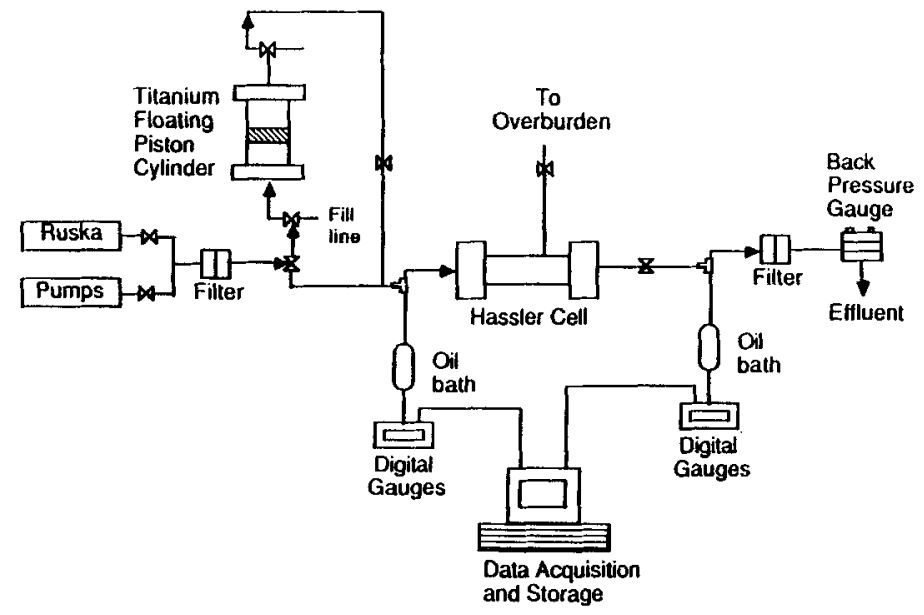

Figure 3. Schematic of coreflood apparatus.

ties. These high velocities prevent precipitate formation (due to the low residence time) and finally cause breakthrough.

Relatively high flow rates (i.e., low Damköhler numbers) result in the formation and deposition of the precipitate much farther down the core. The formation of precipitate farther downstream, combined with the faster wormhole growth rate, results in the formation of a fewer number of "precipitation zones" in the finite length core thus causing fewer fluctuations. For the $5.33 \times 10^{-8} \mathrm{~m}^{3} / \mathrm{s}$ run, the flow rate is relatively high and there is insufficient time for deposition to occur within the core.

A comparison between a run using $0.1 \mathrm{~N} \mathrm{HCl}$ and another using $0.75 \mathrm{~N}$ ferric chloride ( $\mathrm{pH}=1.0$ ) is shown in Figure 5. Both experiments were performed at $1.1 \times 10^{-8} \mathrm{~m}^{3} / \mathrm{s}$ on Texas
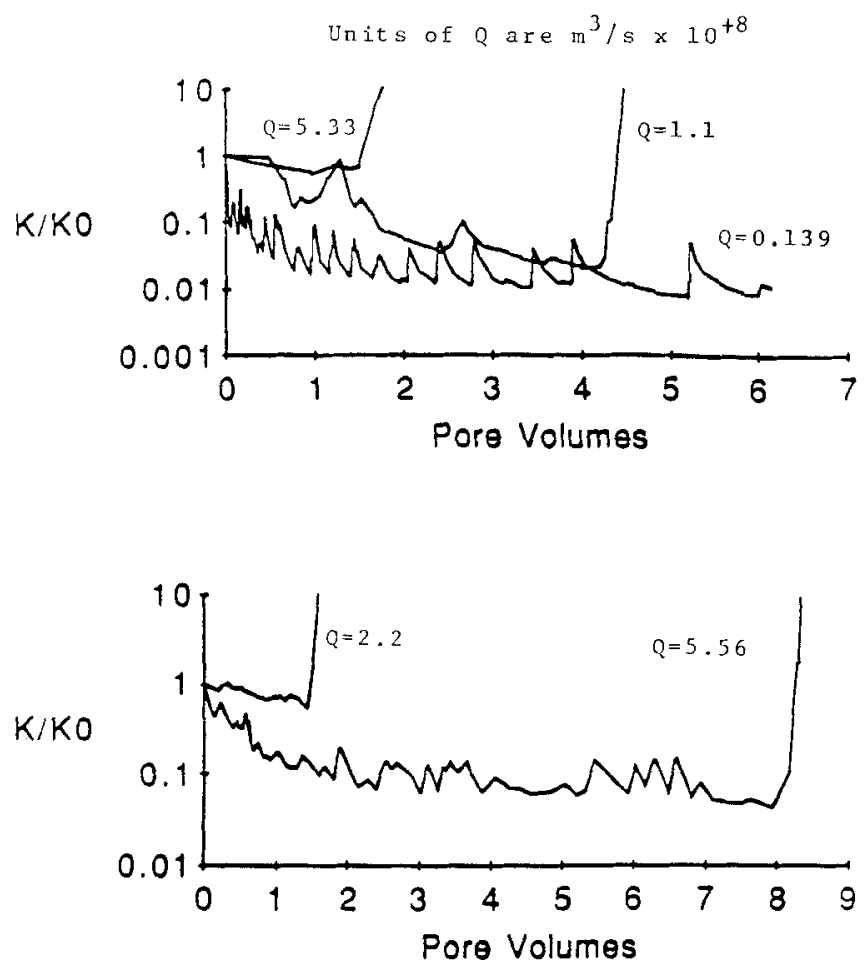

Figure 4. Permeability response for various injection flow rates for ferric chloride/carbonate system in $0.089 \mathrm{~m}$-long limestone cores.
Cream cores. One observes that for $\mathrm{HCl}$ injection the permeability increases steadily in contrast to the initial permeability decline (with fluctuations) for ferric chloride injection. One also observes that the ferric chloride run breaks through in fewer pore volumes because it has a higher acid strength, i.e., $0.75 \mathrm{~mol}$ of ferric chloride can combine with water to yield $2.25 \mathrm{~mol}$ of $\mathrm{H}^{+}$. The higher acid strength results in a faster breakthrough of the dissolution front.

\section{Characterization of the evolved pore structure}

Evolution of the pore structure was characterized using a Wood's alloy casting technique (Hoefner and Fogler, 1987). In this technique, Wood's alloy (a low melting alloy) is imbibed into the core under pressure of one atmosphere at the end of a run. The imbibition pressure is selected so as to ensure that the alloy will only fill up the pore spaces enlarged by dissolution. After the alloy has hardened, the porous matrix is dissolved and the wormhole casting can be observed. The structural difference between an $\mathrm{HCl}$ run and a ferric chloride run at $1.1 \times 10^{-8} \mathrm{~m}^{3} / \mathrm{s}$ is evident from the castings shown in Figure 6. While the $\mathrm{HCl}$ run shows a single dominant wormhole the ferric chloride run is highly branched. Castings of the wormholes for two other flow rates are shown in Figure 7.

\section{Development of Model}

A network model is used to represent the highly interconnected porous formation. The bonds in the network represent the $\mathrm{pH}$ of injected solution $=1.0$

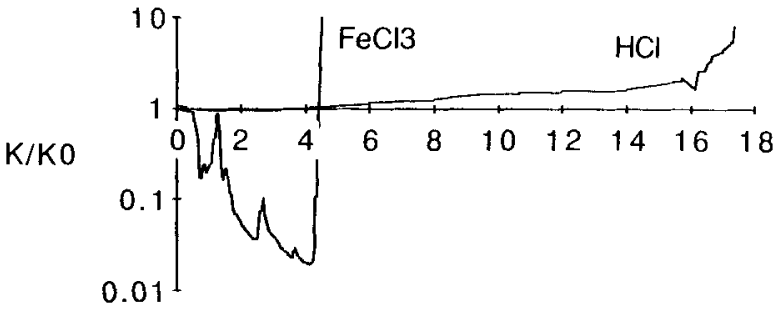

Pore Volumes

Figure 5. Comparison of permeability response of ferric chloride and $\mathrm{HCl}$ runs. 
pore throats and the nodes represent points of mixing. The distribution of sizes assigned to the bonds is identical to the pore throat size distribution of the porous media being studied. While the Voronoi type of network, due to its random nature, most closely represents the porous medium, the regular triangular network has been shown (Jerauld et al., 1984) to possess certain properties (such as conductivity and average coordination number) similar to the Voronoi lattice. Since flow calculations can be performed more efficiently on the regular triangular network as compared to the Voronoi, and good results have previously been obtained using triangular networks (Imadakm and Sahimi, 1987; Rege and Fogler, 1987, 1988) the choice of triangular network was made for this study.

Though the network has previously been used to study several processes in porous media, its use in modeling flow and reaction is fairly recent. Hoefner and Fogler (1988), and later Daccord et al. (1989), have simulated the channel (wormhole) growth during matrix acidizing of limestones using a triangular network model. The bonds in the network are considered to be differential reactors, in which the fluid and the formation minerals interact. Dissolution of the bonds leads to an evolution of the pore structure to form wormholes similar to those observed in core flood experiments.

Reprecipitation of certain minerals complicates the problem considerably. Precipitate of the crystalline form can flow with the fluid and grow with time before depositing, while amorphous precipitate forms a uniform layer on the pore throats. While uniform deposition is mathematically easier to simulate, our previous studies with inert particles flowing through a triangular network have given us a better understanding of the plugging phenomenon due to particle flow in porous media (Rege and Fogler, 1987, 1988).

The current model differs from that of Hoefner and Fogler (1988), in that a "microelement" approach was used. The fluid volume entering the network during a time step is further divided into a large number of "microelements" that maintain their physical identity as they pass through the network. The "microelements" react with the walls of the bonds through which they pass. On arriving at a node the microelements mix

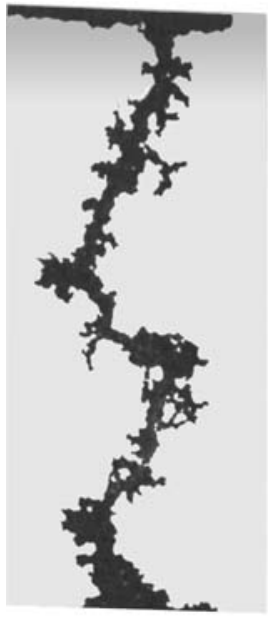

a) $\mathrm{HCl} \mathrm{run}$

$\left(1,1 \times 10^{-8} \mathrm{~m}^{3} / \mathrm{s}\right)$

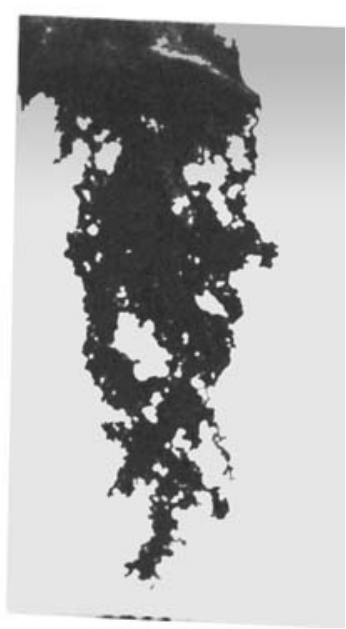

b) $\mathrm{FeCl}_{3} \quad \mathrm{run}$

(1. $\left.1 \times 10^{-8} \mathrm{~m}^{3} / \mathrm{s}\right)$
Figure 6. Comparison of $\mathrm{HCl}$ and ferric chloride castings.

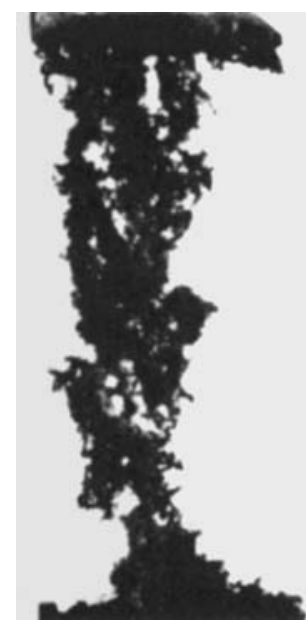

a) $5.33 \times 10^{-8} \mathrm{~min}^{3} / \mathrm{s}$

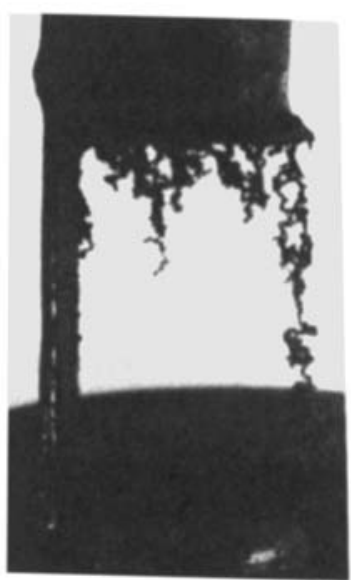

b) $1.39 \times 10^{-9} \mathrm{~m}^{3} / \mathrm{s}$
Figure 7. Wood's metal castings of ferric chloride runs at different flow conditions.

and then flow through the bond selected by flow-biased probability (Rege and Fogler, 1987, 1988). When a sufficiently large number of "microelements" are considered, the concept of "flow-biasing" represents flow being distributed proportionally to the conductance of the bonds present at the node.

The change in the concentration of aqueous species within the "microelement" is then related to the amount of mineral dissolution from the walls (based on the stoichiometric coefficient and the ratio of bond volume to the microelement volume). These changes can then be related to increases or decreases in the bond diameter, which then affect the overall permeability of the network.

The current model is well suited to simulate dissolution, precipitation, and entrapment of particles in porous media. The model can predict the concentration profiles of the aqueous and mineral species for both low and rapid reaction rates. Furthermore, the net changes in the permeability, due to the above processes, can be determined without the use of an empirical relationship. A limitation of the network model is that the network bond volume needs to be scaled with respect to the actual core volume used in the experiment; this scaling parameter can be determined by performing a single experiment.

\section{Results and Discussion}

\section{Case A: equilibrium model}

The current model was first verified for the case of rapid rates of dissolution and precipitation reactions (i.e., an equilibrium model). A simple model system reported by Walsh et al. (1984) was selected for this purpose. In this system, mineral $A B$ dissolves when a fluid containing $A, C$, and $D$ is injected into the porous medium. Solids $A C$ and $B D$ can precipitate when their solubility product is exceeded. Results obtained using the current model for rapid reaction rates were compared with those reported by Walsh et al. (1984).

From Figure 8 we observe that the current network model (symbols) with high rates of reaction (order of $10^{10} \mathrm{cgs}$ units) can indeed reproduce the results of the equilibrium model (bold lines). The $y$-axis represents dimensionless distance and the $x$ axis dimensionless time, i.e., the ratio of the volume of fluid 
injected to the volume of pore space. R I-R 5 are regions containing a fixed composition of species. While the "equilibrium" model can simulate only rapid reaction rates (i.e., equilibrium conditions) the current model can consider the entire spectrum of reaction rates. The network model is very useful in generating concentration surface plots for a range of reaction rates. An example of such a plot is shown in Figure 9 where $C_{p p t}$ represents the concentration of precipitate, $k_{p}$ the rate constant for precipitation, and $x / L$ the dimensionless length along the core. This plot was generated after 1.0 pore volume of fluid had been injected for the example reported by Walsh et al. (1984, see Figure 2) with four chemical species dissolving and only one mineral precipitating. The dissolution reactions are considered to occur instantaneously (rate constant of $10^{10} \mathrm{cgs}$ units), while the rates of precipitation are varied. The rates of precipitation are assumed to be first-order w.r.t. to the degree of supersaturation and the precipitation rate constant, $k_{p}$, is varied from zero to $10^{6} / \mathrm{s}$. We observe that high rates of precipitation result in large amount of localized deposition while moderate rates result in widely distributed deposition.

\section{Case B: dissolution in sandstones}

Dissolution of sandstones using $\mathrm{HF} / \mathrm{HCl}$ acid mixtures has been extensively studied by Fogler and coworkers (Lund and Fogler, 1976; Hekim and Fogler, 1980) using continuum models. In these studies, $\mathrm{HCl} / \mathrm{HF}$ acid mixtures were injected into linear sandstone cores. Input variables such as acid concentration and fluid flow rates were varied, and output variables such as the effluent concentration and the pressure drop across the core were monitored.

Sandstone acidizing was simulated using the current network model. Values of physical constants were identical to those used in the sandstone dissolution studies above. A pore size distribution reported by Lund (1974) was assigned randomly to the bonds in the network. It had been shown that acidization affects primarily the macropores (Swift and Fogler, 1977) and only these were used in the simulation. The analytical solution for acid spending developed by Lund and Fogler (1976) was modified and used to determine concentration changes within each microelement.

A comparison between the network model predictions and the experimental data indicates excellent agreement (Figure 10). As mentioned earlier, a single parameter was used to scale the

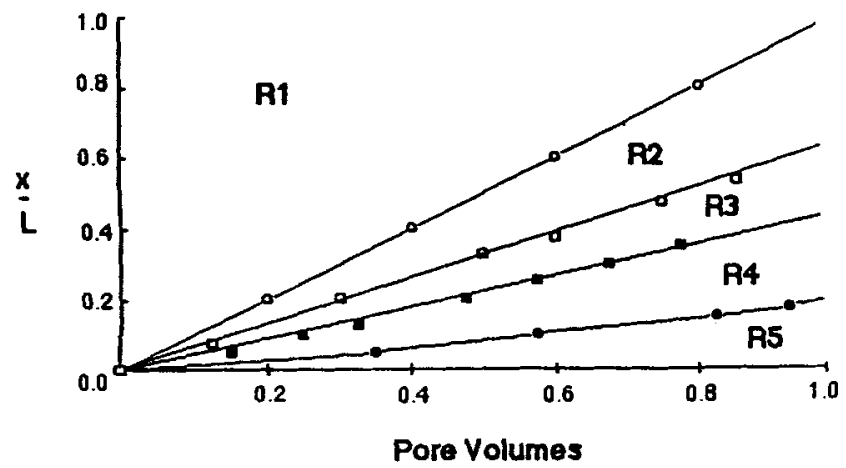

Figure 8. Comparison of current model predictions with that of the "equilibrium" model of Walsh et al. (1984).

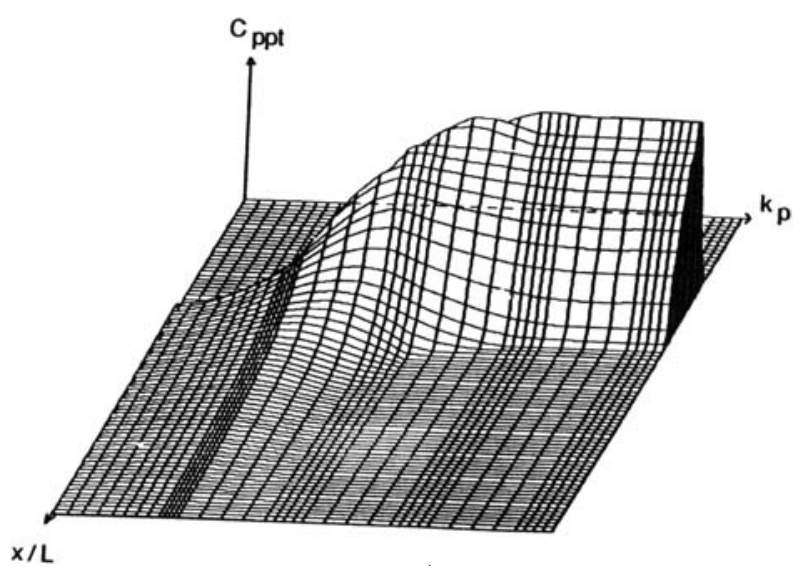

Figure 9. Three dimensional plot of concentration of precipitate as a function of dimensionless distance and dimensionless time.

network pore volumes to the actual pore volume, and a value of 1.2 was used for this example.

A similar study was undertaken on dissolution in carbonates with the "microelement" model. This study gave results identical to those predicted by Hoefner and Fogler (1988), and our results are reported elsewhere (Rege, 1988)

\section{Case $C$ : dissolution and precipitation in carbonates}

Having shown the utility of the microelement method, we then used the network model to simulate the ferric chloride system described earlier. To determine the bond size changes due to reaction, it is necessary to estimate the amount of calcium dissolved in each bond. This amount of calcium dissolved is estimated using the following procedure.

Mass Balance on Calcium (in a bond during an infinitesimal time step)

$$
u \frac{\left.d] \mathrm{Ca}^{2+}\right]}{d x}-\frac{2}{r} R_{\mathrm{Ca}}=0
$$

Rate Law and Stoichiometry

$$
R_{\mathrm{Ca}}=-0.5 R_{H}=0.5 k_{a}\left[\mathrm{H}^{+}\right]
$$

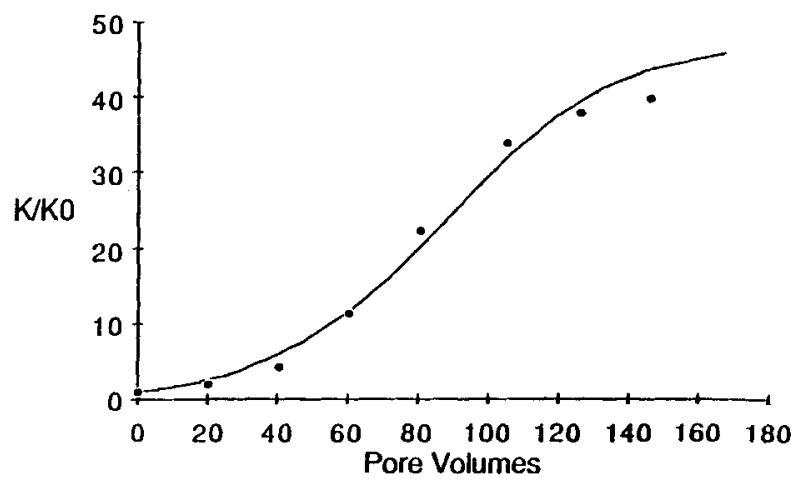

Figure 10. Comparison of model predictions with experimental data for dissolution in sandstones. 
where $k_{a}$ is the mass transfer coefficient having the general form,

$$
k_{a}=a\left(D_{a b}\right)^{2 / 3}(r)^{b-1}(u)^{b}(v)^{1 / 3-b}
$$

(See Appendix C of Hoefner, 1987.)

Choosing the Graetz solution (equivalent to $b=0$ ), and combining Eqs. 4 through 6 one gets,

$$
\frac{d\left[\mathrm{Ca}^{2+}\right]}{\left[\mathrm{H}^{+}\right]}=\frac{\pi a D_{a b}^{2 / 3} \nu^{1 / 3}}{Q} d x
$$

The Damköhler number for dissolution, $\mathrm{Da}_{\mathrm{D}}$, has the form

$$
D a_{D}=a D_{a b}^{2 / 3} L \nu^{1 / 3} / Q
$$

and values range from 0.042 to 1.61 in this study.

In Eq. 7,

$$
\left[\mathrm{H}^{+}\right]=f\left(\left[\mathrm{Ca}^{2+}\right]\right)
$$

For the simple case of hydrochloric acid dissolving carbonate, the functionality, $f$, is given by

$$
\left[\mathrm{H}^{+}\right]=\left[\mathrm{H}^{+}\right]_{0}-2^{*}\left(\left[\mathrm{Ca}^{2+}\right]-\left[\mathrm{Ca}^{2+}\right]_{0}\right)
$$

The functionality for the ferric chloride system is obtained by carrying out mass and charge balances on calcium and ferric ions, i.e., from Figures 1 and 2. These are given below:

$$
\begin{array}{cc}
{\left[\mathrm{H}^{+}\right]=0.1-0.5\left[\mathrm{Ca}^{2+}\right]} & \text { if }\left[\mathrm{Ca}^{2+}\right]<0.1 \\
{\left[\mathrm{H}^{+}\right]=\left\{\left[\mathrm{Fe}^{3+}\right] / K_{s p}\right\}^{1 / 3}} & \text { if }\left[\mathrm{Ca}^{2+}\right]>0.1
\end{array}
$$

where

$$
\left[\mathrm{Fe}^{3+}\right]=0.4-0.45\left[\mathrm{Ca}^{2+}\right] \quad \text { if }\left[\mathrm{Ca}^{2+}\right]>0.1
$$

Typical values of variables appearing in Eq. 5 through $10 \mathrm{~b}$ are:

$$
\begin{gathered}
D_{a b}=4 \times 10^{-9} \mathrm{~m}^{2} / \mathrm{s} \quad \nu=1 \times 10^{-6} \mathrm{~m}^{2} / \mathrm{s} \\
a=1.0 \quad L=0.089 \mathrm{~m}
\end{gathered}
$$

Mean pore throat diameter was $1.0 \mu \mathrm{m}$ (obtained by mercury porosimetry).

Flow rates, $Q$, ranged from $1.39 \times 10^{-9} \mathrm{~m}^{3} / \mathrm{s}$ to $5.33 \times 10^{-8}$ $\mathrm{m}^{3} / \mathrm{s}$.

$\left[\mathrm{H}^{+}\right]_{0}=0.1 \mathrm{~N}$ and $\left[\mathrm{Ca}^{2+}\right]_{0}$

$=0.0 \mathrm{~N}$ at the inlet to the network.

The above functionalities are substituted into Eq. 7 and solved to yield the $\mathrm{Ca}^{2+}$ concentration at the bond exit. The increase in the calcium ion concentration in solution is then related to the increase in the bond diameter.

\section{Flow and precipitation}

The model permits the existence of a supersaturated solution; solid precipitate starts nucleating only after some critical value of supersaturation is reached. [For a 0.75 molar $\mathrm{FeCl}_{3}$ solution, addition of 0.1 molar calcium carbonate results in (calculated from mass and charge balances): $\left[\mathrm{Fe}^{3+}\right] /\left[\mathrm{H}^{+}\right]^{3}=K_{s p}=9.1 \times$ $10^{3}$ (Table 1). Further addition of calcium to the system leads to further depletion of $\mathrm{H}^{+}$ion and $\mathrm{Fe}(\mathrm{OH})_{3}$ starts to form. Though the solubility of $\mathrm{Fe}(\mathrm{OH})_{3}$ is low, it remains in solution till the calcium concentration reaches $0.75 \mathrm{M}$ (supersaturated), and then $\mathrm{Fe}(\mathrm{OH})_{3}$ precipitates out.] This critical value was obtained from batch experiments where it was observed that ferric hydroxide precipitate forms only after a relatively high value of supersaturation has been achieved. After this induction period, precipitate is generated rapidly at a rate, $R_{p}$, given by

$$
R_{p}=k_{p p t}\left[\mathrm{Fe}(\mathrm{OH})_{3} \text { in solution }\right]
$$

The Damköhler number for precipitation, $D a_{p}$, has the form

$$
D a_{p}=k_{p p t} L / u
$$

and is of the order of $10^{10}$, due to rapid rates of precipitation.

Since the precipitation is amorphous in nature, it is laid down uniformly on the bond surface and this deposition increases the resistance to fluid flow.

The above equations are incorporated into the network model and predictions obtained for the permeability response are shown in Figure 11. We observe that the model predicts an increasing number of fluctuations with an increasing Damköhler number. This is in agreement with the trends observed experimentally. For the lowest Damköhler number (highest flow rate), the amount of precipitate deposited in the network was relatively low and caused no damage. Note that for $Q=$ $1.1 \times 10^{-8} \mathrm{~m}^{3} / \mathrm{s}$ units two major and four minor oscillations were observed. An explanation for these fluctuations can be obtained by observing the evolution of the pore structure in a time sequence. Such a time sequence for $Q=1.1 \times 10^{-8} \mathrm{~m}^{3} / \mathrm{s}$ predicted by our model is shown in Figures 12 and 13.

From Figure 12 we observe that initially two channels start to form. At a later stage, one of the wormholes has outgrown the other. Note that certain parts of the channel are plugged up due to the presence of precipitation zones. These precipitation zones prevent further growth of the two initial wormholes. Consequently, most of the fluid is then forced to flow through another region resulting in the formation of a third channel. Since the size of this channel is relatively small and it carries most of the flow, the interstitial velocity in this third channel is high. The high velocity results in a low residence time preventing the formation of the precipitate within the network. This channel eventually breaks through as seen in the last network in Figure 12. These conclusions are confirmed by observing the fluid flow profiles in Figure 13 that were obtained for the same time step as the corresponding ones in Figure 12. The two major fluctuations observed in Figure 11 correspond to the channels being blocked at times $t_{2}$ and $t_{3}$, respectively. The minor fluctuations in Figure 11 for $Q=1.1 \times 10^{-8} \mathrm{~m}^{3} / \mathrm{s}$ are caused by smaller individual branches growing and withering.

\section{Summary and Conclusions}

A network model combined with the "microelement" approach has been developed to describe flow and reaction in porous media. The model can predict the permeability response for both reaction-rate-limited (sandstone acidizing) and mass- 

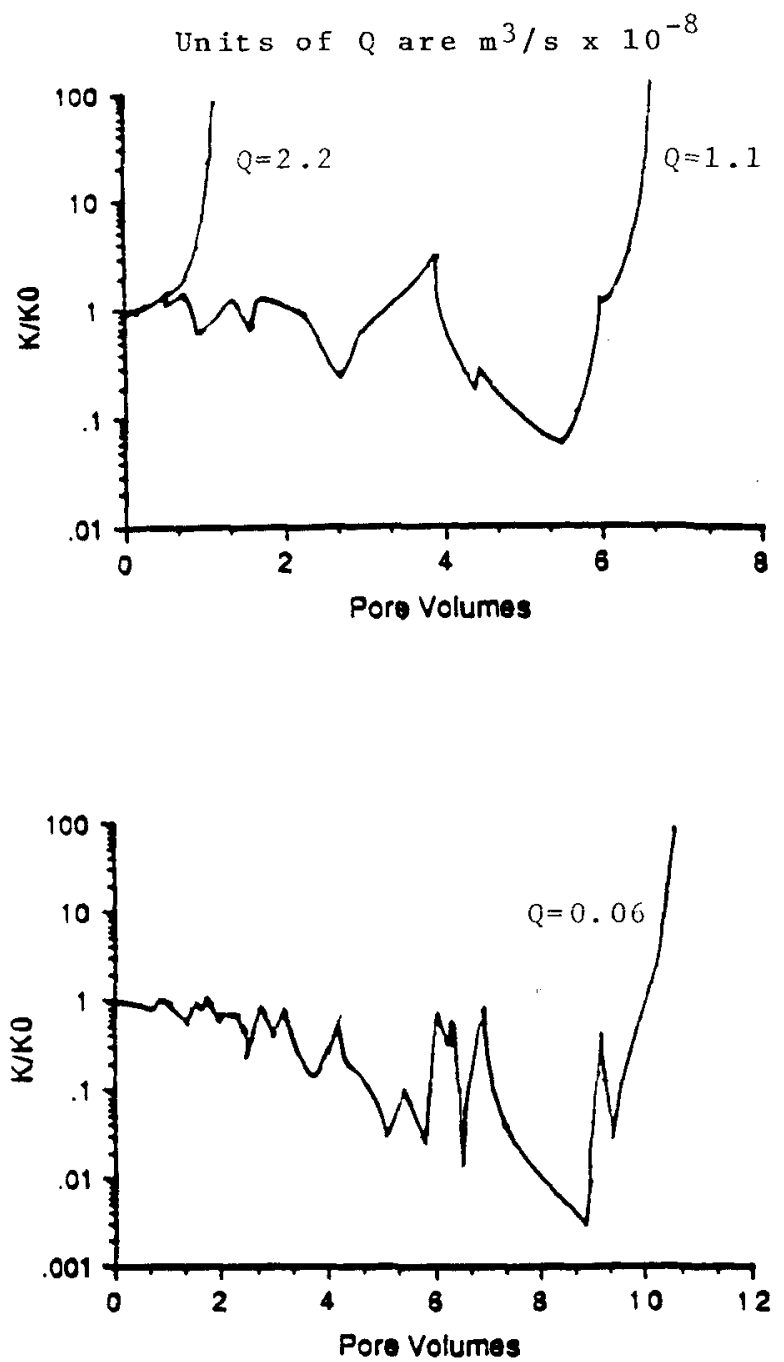

Figure 11. Predictions of the permeability response for various flow rates. Pressure fluctuations predicted are in good agreement with experimental data.

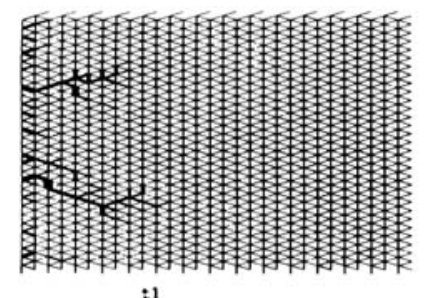

:1

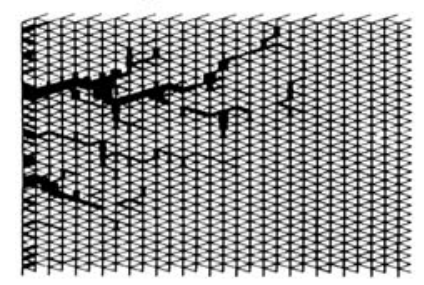

t3

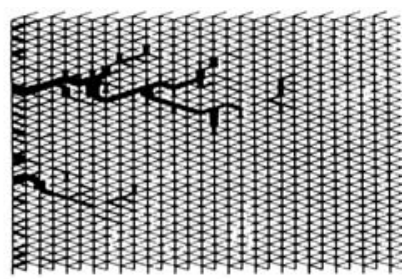

t2

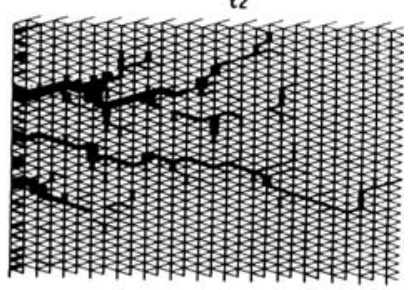

t4
Figure 12. Predicted changes in the bond diameters as a time sequence.

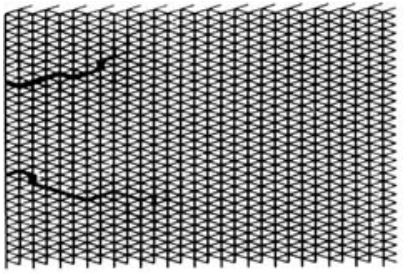

t1

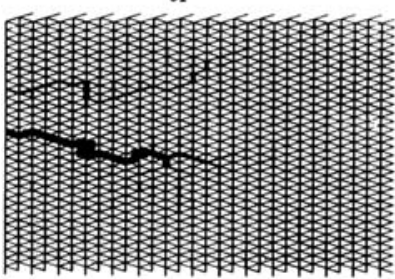

t3

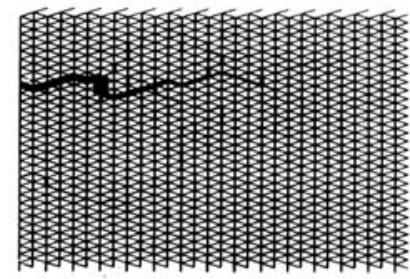

t2

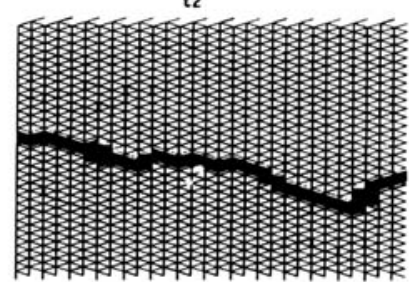

Figure 13. Predicted flow profiles in the network as a time sequence.

transfer-limited reactions (carbonate acidizing). The versatility of the model was demonstrated by predicting concentration waves for rapid reaction rates (i.e., equilibrium conditions); these predictions agreed excellently with existing theory. Experiments were performed with ferric chloride and limestone system to delineate the effects of the Damköhler number on the extent of permeability decline due to precipitation. Unexpected fluctuations were observed in the permeability response and the frequency of these fluctuations was related to the Damköhler number. The current model predicted these trends qualitatively. Time sequences of the pore evolution predicted by the model demonstrated the importance of considering flow redistribution, finite rates of reaction, and the network model when studying competition between dissolution and precipitation in porous media. The model was tested with experimental data and other models; and good agreement was observed in all cases.

\section{Acknowledgment}

The authors gratefully acknowledge the support of the members of our Industrial Affiliates Program on Flow and Reaction in Porous Media at The University of Michigan: Chevron Oil Field Research Co., Conoco Inc., Halliburton Services, Marathon Oil, Mobil R\&D, Japan Oil Co., and Texaco Inc. We also wish to thank the National Science Foundation (grant no. CBT 8516458) for partial support and the Pittsburgh Supercomputing Center for use of their Cray X-MP Supercomputer.

\section{Notation}

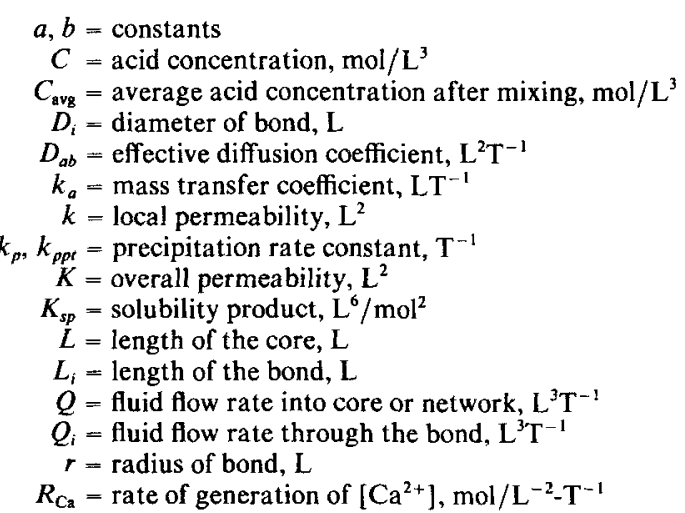


$R_{\mathrm{H}}=$ rate of consumption of $\left[\mathrm{H}^{+}\right], \mathrm{mol} / \mathrm{L}^{-2}-\mathrm{T}^{-1}$

$u=$ velocity, $\mathrm{LT}^{-1}$

\section{Greek letters}

$$
\begin{aligned}
\Delta \phi & =\text { change in porosity } \\
\beta & =\text { parameter } \\
\nu & =\text { kinematic viscosity, } \mathrm{L}^{2} \mathrm{~T}^{-1}
\end{aligned}
$$

\section{Literature Cited}

Axtmann, R. C., and L. B. Peck, "Geothermal Chemical Engineering," AIChE J., 22, 817 (1976)

Butler, J. N., Ionic Equilibrium: A Mathematical Approach, AddisonWesley Publishing, 364 (1964).

Bryant, S. L., R. S. Schechter, and L. W. Lake, "Mineral Sequences in Precipitation/Dissolution Waves," AIChE J., 33, 1271 (1987).

-__ "Interactions of Precipitation/Dissolution Waves and Ion Exchange in Flow Through Permeable Media," AIChE J., 32, 751 (1986).

Crowe, C. W., "Precipitation of Hydrated Silica from Spent Hydrofluoric Acid: How Much of a Problem Is It?,"J. Petr. Technol., 1234, (1986).

Daccord, G., E. Touboul, and R. Lenormand, "Carbonate Acidizing: Toward a Quantitative Model of the Wormholing Phenomenon," SPE Production Eng., 63 (Feb. 1989).

Fogler, H. S., and S. D. Rege, "Porous Dissolution Reactors," Chem. Eng. Commun., 42, 291 (1985).

Hekim, Y., and H. S. Fogler, "On the Movement of Multiple Reaction Zones in Porous Media," AIChE J., 26(3), 403 (May, 1980).

Hoefner, M. L., "Matrix Acidizing in Carbonates using Microemulsions: The Study of Flow, Dissolution, and Channeling in Porous Media," PhD Thesis, Univ. of Michigan (1987).

Hoefner, M. L., and H. S. Fogler, "Effect of Acid Diffusion in Matrix Acidizing in Carbonates," J. Pet. Tech. (1987).

-, "Pore Evolution and Channel Formation during Flow and Reaction in Porous Media," AIChE J., 34, 45 (1988).

Hoffman, M. R., "Brine Chemistry-Scaling and Corrosion," Environ. Quality Lab. Memor., No. 14, 38 (1975).

Hwang, Y., F. G. Helfferich, and Rong-Jin Leu, "Multicomponent Equilibrium Theory for Ion-Exchange Columns Involving Reactions," AIChE J., 34, 1615 (1988).

Imdakm, A. O., and M. Sahimi, "Transport of Large Particles in Flow Through Porous Media," Phys. Rev., A36, 5304 (1987).

Jasti, J. K., J. T. Lindsay, and H. S. Fogler, "Flow Imaging in Porous Media using Neutron Radiography," SPE 16950 (1987).

Jerauld, G. R., J. C. Hatfield, L. E. Scriven, and H. T. Davis, "Percola- tion and Conduction of Voronoi and Triangular Networks: A Case Study in Topological Disorder," J. Phys. C.,17, 3429 (1984).

Lund, K., "On the Acidization of Sandstone," PhD Thesis, Univ. of Michigan (1974).

Lund, K., and H. S. Fogler, "Acidizations: V. The Prediction of the Movement of Acid and Permeability Fronts in Sandstone," Chem. Eng. Sci., 31, 381 (1976).

McMennamy, G., "A Comparison of Iron Contral Techniques," Southwestern Petroleum Short Course.

Novak, C. F., R. S. Schechter, and L. W. Lake, "Rule Based Mineral Sequences in Geochemical Flow Processes," AIChE J., 34, 1607 (1988).

Rege, S. D., "Network Modeling and Experimental Investigation of Flow, Dissolution, Precipitation and Fines Migration in Porous Media," PhD Thesis, Univ. of Michigan (1988).

Rege, S. D., and H. S. Fogler, "A Network Model for Straining Dominated Capture in Porous Media," Chem. Eng. Sci.,42(7), 1553 (1987).

Rege, S. D., and H. S. Fogler, "A Network Model for Deep Bed Filtration of Emulsions and Solids," AIChE J.,accepted for publication (1988).

Reistad, G. M., W. E. Scmisseur, J. R. Shay, and J. B. Fitch, “An Evaluation of Uses for Low to Intermediate Temperature Geothermal Fluids in the Klamuth Basin, Oregon," Oregon State Engineering Experiment Station Bulletin, No. 55 (1978).

Sahimi, M., and T. T. Tsotsis, "Statistical Modeling of Gas-Solid Reaction with Pore Volume Growth: Kinetic Regime," Chem. Eng. Sci., 43, 113 (1988).

"Dynamic Scaling for the Fragmentation of Reactive Porous Media," Phys. Rev. Lett., 59, 888 (1987).

, "A Percolation Model of Catalyst Deactivation by Site Coverage and Pore Blockage," J. Cat., 96, 552 (1985).

Shah, N., and J. M. Ottino, "Transport and Reaction in Evolving, Disordered Composites: II. Coke Deposition in a Catalytic Pellet," Chem. Eng. Sci., 42, 63 (1987).

Smith, C. E., C. E. Crowe, and T. J. Nolan, III, "Secondary Deposition of Iron Compounds Following Acidizing Treatments," SPE 2358 (1968).

Swift, S. T., and H. S. Fogler, "A Note on Capillary Model Developments for Sandstone Acidization," Chem. Eng. Sci.,32, 339 (1977).

Too, J. R., L. T. Fan, and R. Nassar, "A Stochastic Axial Dispersion Model for Tubular Flow Reactors," Chem. Eng. Sci., 41, 2341 (1986).

Walsh, M. P., S. L. Bryant, R. S. Schechter, and L. W. Lake, "Precipitation and Dissolution of Solids Attending Flow through Porous Media," AIChE J., 30(2), 317 (1984).

Manuscript received Dec. 27, 1988, and revision received Mar. 16, 1989. 